\title{
Il calcolo urinario. Proposta di un nuovo metodo di determinazione: dati preliminari
}

\author{
Giovanni Cangiano¹, Grazia Buccino ${ }^{1}$, Annachiara Latte ${ }^{2}$, Marianna Bencivenga ${ }^{3}$, Giovanna Capolongo ${ }^{4}$, Angela Lamanna ${ }^{5}$, \\ Marco Terribile ${ }^{6}$, Claudia Passaretti ${ }^{7}$, Maurizio D’Amora7
}

\author{
${ }^{1}$ Laboratorio di Patologa Clinica P.O. dei Pellegrini ASL NA 1 Centro, Napoli - Italia \\ ${ }^{2}$ Sevizio di Farmacia, Ospedale Santa Chiara, Trento - Italia \\ ${ }^{3}$ Nefrologia e Dialisi, P.O. dei Pellegrini ASL NA 1 Centro, Napoli - Italia \\ ${ }^{4}$ Nefrologia e Dialisi, Ospedale del Mare ASL NA 1 Centro, Napoli - Italia \\ ${ }^{5}$ Pediatria, Università della Campania Luigi Vanvitelli, Napoli - Italia \\ ${ }^{6}$ Urologia, Università della Campania Luigi Vanvitelli, Napoli - Italia \\ ${ }^{7}$ Medicina di Laboratorio, P.O. San Paolo ASL NA 1 Centro, Napoli - Italia
}

\begin{abstract}
Urinary calculus. A new method of determination: preliminary data
Introduction: The visualization of reactions used for the identification and quantification of substances in the urinary calculus presents significant problems.

Methods: We describe a new laboratory procedure for the determination of the urinary calculus. The detection of carbonates and the concentrations of calcium, phosphorus, magnesium, ammonium, uric acid (quantitative dosages carried out on COBAS 6000 analyzer of the Roche company) and oxalate and cystine (determinations adapted by us on the Siemens Viva E instrument) are entered in an Excel sheet to obtain the main possible urinary calculations (calcium oxalate, uric acid, ammonium urate, cystine, struvite, brushite, apatite, carbonate apatite) and more generally "calcium phosphate". The clinical chemistry determinations are similar to those used for the metabolic study carried out on the $24 \mathrm{~h}$-urine in which the photometric dosage of cystine with phosphotungstic acid, not being marketed, was built and adapted on Viva E analyzer. The use of the rapid quantitative method, with non-commercial sulfosalicylic acid, built in our laboratory, is particularly useful for the determination of oxalate in the calculation.

Results: The software proposed converts all concentrations of the above mentioned analytes to mmoles/dL and, starting from struvite, the possible presence of calcium oxalate, ammonium urate and different calcium phosphate, determined according to the $\mathrm{Ca} / \mathrm{P}$ ratio.

Conclusions: The data obtained, especially if referring to concretions formed by several components, show good results when compared with the FTIR technique.
\end{abstract}

Keywords: Excel, FTIR, Urinary calculus

\section{Introduzione}

Il Laboratorio del P.O. dei Pellegrini ha da tempo abbandonato il metodo chimico per la determinazione della composizione del calcolo urinario. Con tale metodologia si effettua,

Received: May 25, 2020

Accepted: June 3, 2020

Published online: June 27, 2020

Indirizzo per la corrispondenza:

Giovanni Cangiano

Laboratorio di Patologia Clinica

P.O. dei Pellegrini - ASL NA 1 Centro

Via Portamedina

80134 Napoli - Italia

gianni.cangiano@libero.it infatti, una valutazione visiva dei colori scaturiti dalle sette diverse reazioni proposte nel kit e, precisamente, per la rilevazione di calcio, fosforo, magnesio, acido urico, ammonio, cistina e ossalato. Si determinano, inoltre, i carbonati con acido solforico e, infine, si estrapolano le principali componenti del calcolo in questione grazie all'uso di uno specifico regolo fornito dalla ditta produttrice (1-3).

I notevoli problemi derivanti dalla visualizzazione soggettiva delle diverse reazioni e dall'identificazione e dalla quantificazione delle diverse sostanze presenti nel calcolo urinario vengono da noi risolti proponendo una metodica di laboratorio pratica, rapida e affidabile.

In un'analisi preliminare (febbraio-ottobre 2019: 58 calcoli urinari/renali), si confrontano i dati ottenuti con la nostra nuova proposta biochimica rispetto a quelli rilevati usando la tecnica di riferimento all'infrarosso. 


\section{Materiali e metodi}

\section{Preparazione del campione e dosaggio di calcio, fosforo, magnesio, ammonio e carbonati}

$10 \mathrm{mg}$ di calcolo frantumato su mortaio in agata sono trattati con $125 \mu \mathrm{L}$ di acido solforico concentrato; dopo completa dissoluzione al vortex, si aggiungono $10 \mathrm{~mL}$ di acqua distillata. Dopo miscelazione e successiva centrifugazione, il surnatante viene sottoposto ad analisi di routine per la determinazione di calcio, fosforo, magnesio, acido urico e ammonio (Cobas 6000 Roche) e cistina e ossalato (Viva E Siemens). A parte si ricercano i carbonati osservando l'eventuale effervescenza dopo trattamento del calcolo con acido solforico concentrato (4-6).

Il surnatante viene sottoposto a diluizione automatica di 1:3 per l'analisi del calcio e di 1:20 per quella dell'ammonio; è, inoltre, indispensabile determinare un bianco per l'ammonio su una soluzione costituita da $125 \mu \mathrm{L}$ di acido solforico concentrato aggiunti a $10 \mathrm{~mL}$ di acqua distillata.

\section{Determinazione della cistina}

La metodica, da noi costruita e adattata sullo strumento Viva E della ditta Siemens a tre reagenti, è l'unica metodica che presenta delle particolarità analitiche meritevoli di una maggiore attenzione. Essa viene, pertanto, così rappresentata nei seguenti step:

1) l'ago reagenti preleva $220 \mu \mathrm{L}$ di soluzione R1 (10 acetato di sodio tri-idratato $2 \mathrm{M}$ : 2 acido acetico $2 \mathrm{M}$ : 8,4 acqua). Segue un lavaggio con $\mathrm{HCl} \mathrm{0,1} \mathrm{M;}$

2) dopo circa 2 minuti l'ago campione preleva $30 \mu \mathrm{L}$ di soluzione del calcolo (o di urina);

3) dopo altri 2 minuti di attesa, l'ago reagenti preleva $30 \mu \mathrm{L}$ di soluzione R2 (solfito di sodio 0,1 M, stabile 1 mese in frigo). Segue un lavaggio con $\mathrm{HCl} \mathrm{0,1} \mathrm{M;}$

4) è necessaria un'ulteriore attesa di circa 2 minuti prima che l'ago reagenti prelevi $120 \mu \mathrm{L}$ di soluzione R3 (acido fosfotungtico $30 \%$, stabile indefinitamente in frigo). Dopo circa 6' 30" di attesa, si rileva la concentrazione di cistina, in $\mathrm{mg} / \mathrm{dL}$, previa misurazione fotometrica a $660 \mathrm{~nm}$ (formazione di blu di tungsteno). La calibrazione a 6 punti (da 0 a $40 \mathrm{mg} / \mathrm{dL}$ ) si riferisce a una spline cubica modificata;

5) la determinazione del "bianco campione" si effettua sostituendo la soluzione R1 con una nuova, effettuata al momento e composta da 3,3 $\mathrm{mL}$ della precedente (tampone acetato e acqua) e $27 \mu \mathrm{L}$ di cloruro mercurico 0,1 $\mathrm{M}$. La concentrazione di cistina si ricava rieseguendo il test e, successivamente, sottraendo il valore ottenuto a quello determinato in precedenza (7-14).

\section{Determinazione dell'ossalato}

\section{a. Ossalato Trinder}

La determinazione Trinder (ditta LTA) necessita di una diluizione 1:10 della soluzione del calcolo con tampone EDTA
$\mathrm{pH}$ 7,0. La metodica manuale viene adattata sull'analizzatore Viva E e prevede i seguenti punti:

1) l'ago reagenti preleva $220 \mu \mathrm{L}$ di soluzione $\mathrm{R} 1$ (tampone succinato $\mathrm{pH} 3$,8/acido 3 dimetil-amino benzoico (DMAB)/ conservanti/stabilizzanti): il reattivo 1 viene miscelato in parti uguali con il reattivo 2 a seconda del numero di determinazioni;

2) dopo circa 2 minuti, l'ago campione preleva $9 \mu \mathrm{L}$ di soluzione del calcolo (o di urina);

3) dopo altri 4 minuti di attesa, l'ago reagenti preleva $50 \mu \mathrm{L}$ di soluzione R3 (reattivo 2: ossalato ossidasi/POD/conservanti/stabilizzanti. Il liofilo viene disciolto con $5 \mathrm{~mL}$ di acqua: stabile 1 mese in congelatore);

4) dopo circa 6' 30" di attesa, si rileva la concentrazione di ossalato, in $\mathrm{mmol} / \mathrm{L}$, previa misurazione fotometrica a 600 $\mathrm{nm}$ (formazione di indamina). La reazione a termine viene quantificata con uno standard di 0,50 mmol/L e l'utilizzo di un bianco campione. Si utilizzano anche 2 soluzioni di controlli a concentrazione di 0,25 e 1,00 mmol/L (15-18).

\section{b. Ossalato al ferro-solfosalicilico}

Il principio biochimico si basa sull'azione dell'ossalato che decolora il complesso ferro-solfosalicilico in ambiente tamponato (borato) ed evidenziato alla lunghezza d'onda di $505 \mathrm{~nm}$.

$10 \mathrm{mg}$ di calcolo frantumato sono trattati con $125 \mu \mathrm{L}$ di acido solforico concentrato; dopo completa dissoluzione al vortex, si aggiungono $10 \mathrm{~mL}$ di acqua distillata. Dopo miscelazione e successiva centrifugazione, il sopra natante ottenuto si dosa sulla strumentazione biochimica succitata. A $220 \mu \mathrm{L} \mathrm{di}$ tampone borato $(0,1 \mathrm{M} \mathrm{pH} 9,5)$ l'analizzatore aggiunge $30 \mu \mathrm{L}$ di campione. Successivamente sono aggiunti $21 \mu \mathrm{L}$ di cloruro ferrico esa-idratato $(0,09 \mathrm{~g} / \mathrm{dL})$ e $21 \mu \mathrm{L}$ di acido solfosalicilico $(1 \mathrm{~g} / \mathrm{dL})$. Dopo un'attesa di circa 6,5 minuti si ricava la concentrazione dell'analita riferendoci a una curva di calibrazione a 6 punti (modello spline cubica modificata) compresi tra 0,00 e $6,00 \mathrm{mmol} / \mathrm{L}$ di ossalato di sodio $(0,00-0,50-1,00-2,00-4,00$ e 6,00 ) e ricavati da una soluzione madre di $16 \mathrm{mmol} / \mathrm{L}$. Si utilizzano anche 2 soluzioni di controlli a concentrazione di 1,50 e 5,00 mmol/L. Anche le soluzioni dei calibratori e dei controlli sono state costituite con l'aggiunta di acido solforico concentrato (19).

\section{Determinazione del calcolo all'infrarosso}

Si fa uso di uno spettrofotometro FTIR (Fourier Transform Infrared) della ditta Shimadzu. I campioni vengono preparati miscelando, in un mortaio in agata, circa $200 \mathrm{mg}$ di bromuro di potassio anidro (almeno 12 ore in stufa a $150^{\circ} \mathrm{C}$ ) con circa $2 \mathrm{mg}$ di calcolo precedentemente frantumato. Un sistema pastigliatore, alla pressione di circa $7 \mathrm{~atm}$ e per un tempo di 5-6 min, ricava un dischetto da inserire nell'alloggio campionatore del FTIR. 
La misurazione dello spettro del campione viene preceduta da quella effettuata su dischetto di bromuro di potassio (bianco campione). L'analisi e la rilevazione/comparazione degli spettri avviene grazie a uno specifico software (6-20).

\section{Elaborazione in Excel}

Si fa uso di un opportuno software non commerciale, da noi ideato, che estrapola le più comuni componenti di un calcolo urinario quali struvite, calcio ossalato, acido urico e urato di ammonio, calcio fosfato in generale, ma, anche, brushite, apatite e carbonato apatite. Dalle concentrazioni iniziali dei sette analiti dosati è possibile ricavare i costituenti del calcolo, espressi in percentuale $(6,21,22)$.

\section{II foglio elettronico}

1) Immissione dati e percentualizzazione analiti chimici: si effettua nelle celle E4-E10 (Fig. 1): calcio, fosforo, acido urico, cistina e magnesio sono espressi in $\mathrm{mg} / \mathrm{dL}$, l'ossalato è espresso in $\mathrm{mmol} / \mathrm{L} \mathrm{e}$, infine, l'ammonio in $\mu \mathrm{g} / \mathrm{dL}$.
Verranno inseriti nel calcolo solamente valori superiori alle rispettive soglie elencate nelle celle G4-G10: concentrazioni inferiori o uguali al proprio valore soglia saranno considerate equivalenti a 0 . Le nuove concentrazioni espresse in $\mathrm{mg} / \mathrm{dL}$ verranno evidenziate nelle celle F4-F10; la somma di tali concentrazioni trovate sarà visualizzata in F11. La percentuale di ogni elemento trovato viene, quindi, calcolata nelle celle I4-I10 (p. es., \% Ca --> $14=\mathrm{F} 4 * 100 / \mathrm{F} 11)$.

2) Trasformazione in $\mu \mathrm{mol} / \mathrm{dL}$ : si effettua nelle celle $\mathrm{H} 4-\mathrm{H} 9$. Nel caso del calcio, la concentrazione in $\mathrm{mg} / \mathrm{dL}$ evidenziata in $\mathrm{F} 4(26,80 \mathrm{mg} / \mathrm{dL}$ ), se moltiplicata per 1000 (passaggio da $\mathrm{mg}$ a $\mu \mathrm{g}$ ) e divisa per 40 (PM del calcio) si trasforma in un valore espresso in $\mu \mathrm{mol} / \mathrm{dL}$ e visualizzato in $\mathrm{H} 4(670,0$ $\mu \mathrm{mol} / \mathrm{dL}$ ). Procedimento simile si effettua per gli altri elementi del calcolo, considerando i relativi $\mathrm{PM}(\mathrm{P}=31 ; \mathrm{Mg}=$ 24,3; NH4 = 18; Ox = 88; Ac. urico = 168,1).

3) Quantificazione della struvite (MgNH4PO4.6H2O: PM $=245,4)$ : si confrontano le concentrazioni di fosforo (H5), magnesio $(\mathrm{H} 6)$ e $\mathrm{NH} 4(\mathrm{H} 7)$ presenti nella struvite (Fig. 2). Essendo i tre componenti presenti in quantità

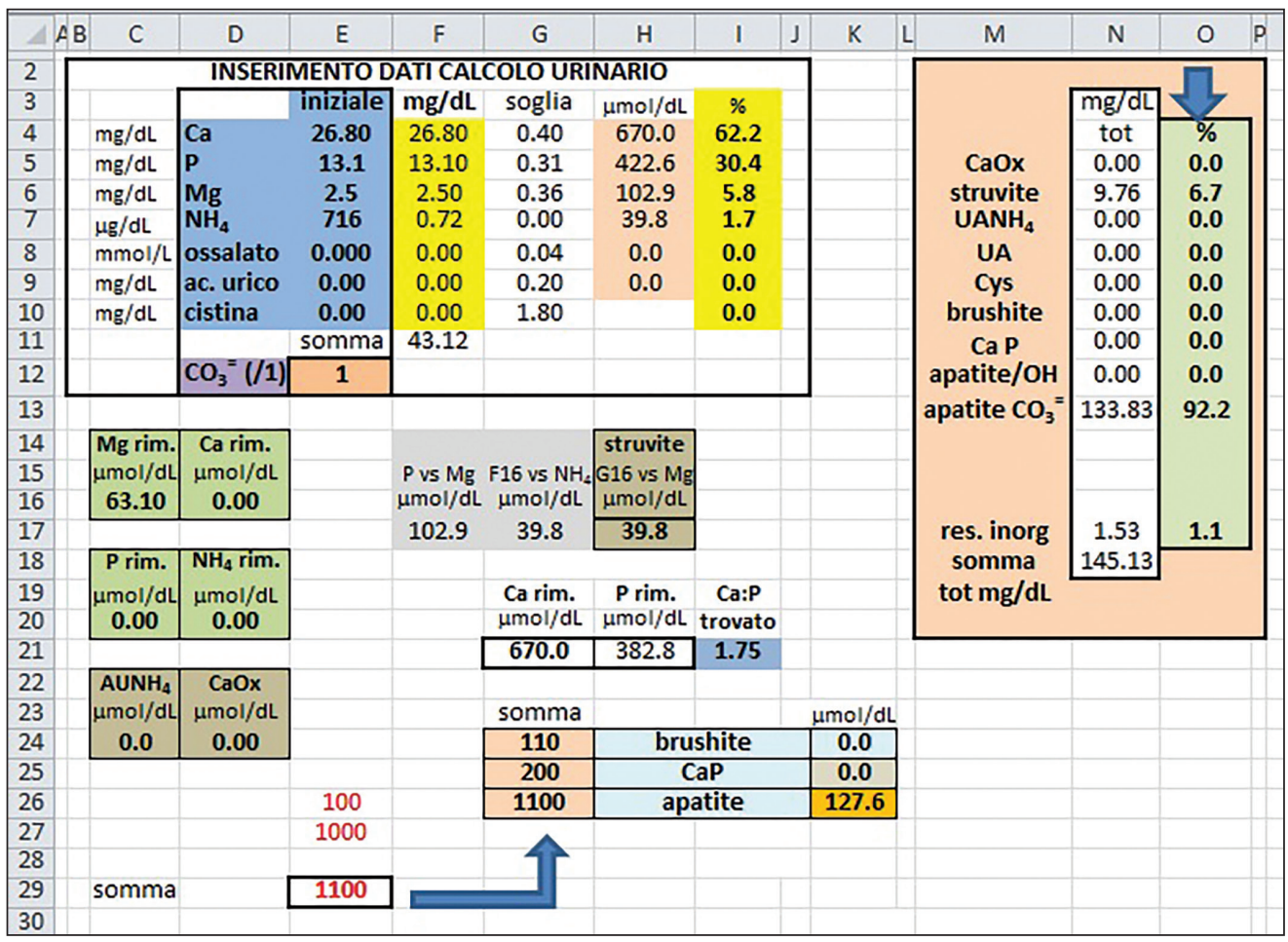

Fig. 1 - Foglio elettronico per esame del calcolo urinario/renale

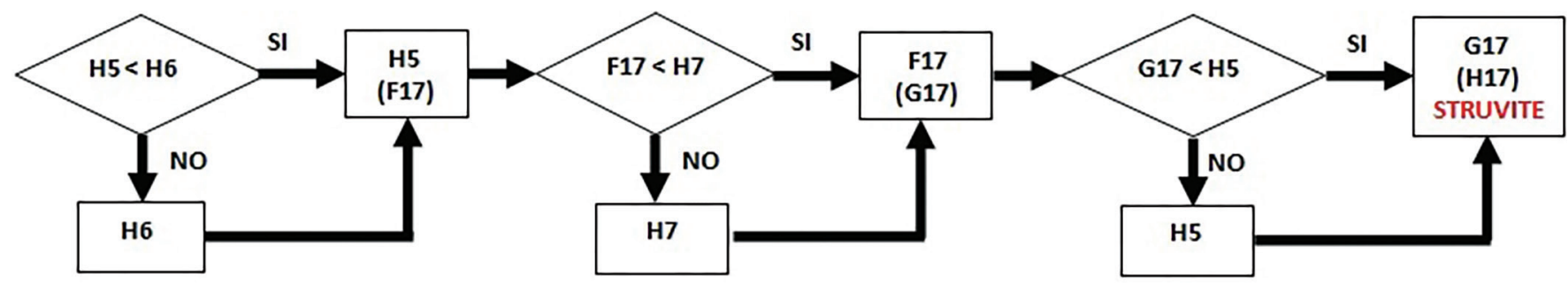

Fig. 2 - Diagramma di flusso per la ricerca della struvite 


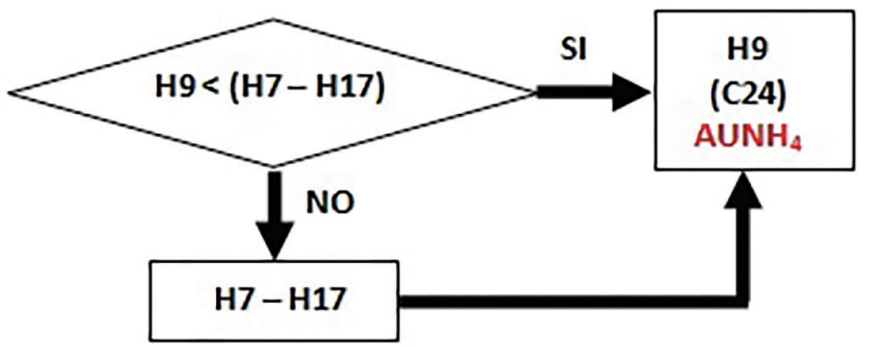

Fig. 3 - Diagramma di flusso per la ricerca dell'urato di ammonio

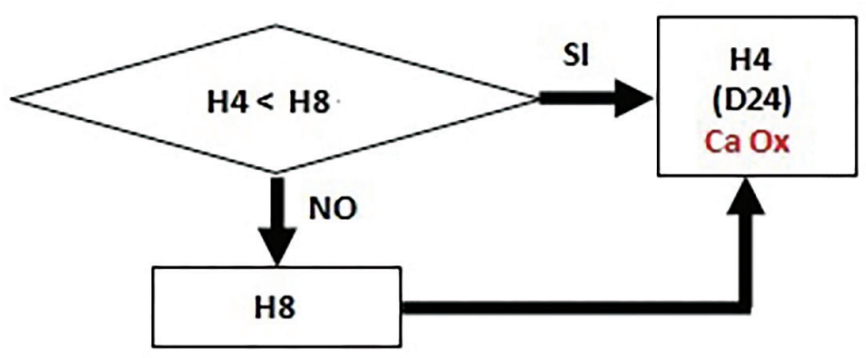

Fig. 4 - Diagramma di flusso per la ricerca di calcio ossalato

equimolecolare (espressa sempre in $\mu \mathrm{mol} / \mathrm{dL}$ ), nella cella F17 si ricava il valore più piccolo tra H5 $(422,6)$ e H6 $(102,9)$ e precisamente $102,9 \mu \mathrm{mol} / \mathrm{dL}$. Conseguentemente, in G17 si evidenzia il valore più piccolo tra F17 $(102,9)$ e $H 7(39,8)$, ossia 39,8. La quantità di struvite, espressa in $\mu \mathrm{mol} / \mathrm{dL}$, è rappresentata in $\mathrm{H} 17$ (si estrapola il valore più piccolo per confronto tra G17 [39,8] e H5 [422,6]) $\rightarrow 39,8 \mu \mathrm{mol} / \mathrm{dL}$.

4) Quantificazione dell'urato di ammonio (AUNH4: $P M=$ 185,1): si confrontano le concentrazioni di acido urico (H9) e NH4 rimanente (H7-H17 "struvite") (Fig. 3). Essendo i due componenti presenti in quantità equimolecolare (espressi sempre in $\mu \mathrm{mol} / \mathrm{dL}$ ), nella cella $\mathrm{C} 24$ si ricava il valore più piccolo tra $\mathrm{H} 9(0,0)$ e $\mathrm{H} 7-\mathrm{H} 17$ e, precisamente, $0,0 \mu \mathrm{mol} / \mathrm{dL}$.

5) Quantificazione dell'ossalato di calcio (CaOx. H2O:PM = 146,1): si confrontano le concentrazioni di calcio (H4) e ossalato (H8) (Fig. 4). Essendo i due componenti presenti in quantità equimolecolare (espressi sempre in $\mu \mathrm{mol} /$ dL), nella cella D24 si confrontano i valori di H4 $(670,0)$ e $\mathrm{H} 8(0,0)$ : se la differenza tra $\mathrm{H} 4$ e $\mathrm{H} 8$ è negativa, il calcio ossalato assume il valore di $\mathrm{H} 4(0,0 \mu \mathrm{mol} / \mathrm{dL})$, altrimenti il valore di $\mathrm{H} 8(0,0 \mu \mathrm{mol} / \mathrm{dL})$.

6) Rapporto $\mathrm{Ca} / \mathrm{P}$ : nella cella $\mathrm{G} 21$ viene rappresentato il calcio rimanente, espresso in $\mu \mathrm{mol} / \mathrm{dL}$, rappresentato dalla differenza tra il calcio (H4) e il calcio ossalato (D24) equivalente a 670,0; nella cella H21 il fosforo rimanente è, invece, calcolato con la differenza tra il fosforo iniziale e quello presente nella struvite (H5-H17): $382,8 \mu \mathrm{mol} / \mathrm{dL}$. Nella cella $121 \mathrm{si}$ rapportano le concentrazione di Ca e P rimanenti (G21/ $\mathrm{H} 21$ ): il valore risultante è di 1,75 . Nei casi in cui è assente il fosforo (si otterrebbe un rapporto con denominatore 0 ), la cella I21 assume il valore di 1000000 (Fig. 5).

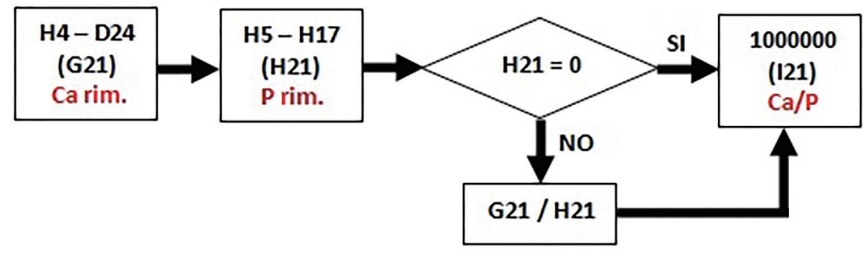

Fig. 5 - Diagramma di flusso per il rapporto $\mathrm{Ca} / \mathrm{P}$

7) Identificazione di calcio fosfato e sua quantificazione: i valori di $\mathrm{Ca} / \mathrm{P}$ da noi impostati in Excel sono compresi tra $\mathrm{i}$ seguenti range:

brushite $<1,155 ; 1,155 \leq$ Ca $\mathrm{P}<1,450$; apatite $\geq 1,450$.

Con opportuni confronti e punteggi (brushite $=10 ; \mathrm{Ca}$ $P=100$ e apatite $=1000$ ), la somma finale ottenuta caratterizza il tipo di calcio fosfato presente nel calcolo.

$\mathrm{Nel}$ nostro caso, dato che il valore trovato di Ca/P (I21), equivalente a 1,75 , è maggiore di 1,155 , allora il valore della cella E26 sarà equivalente a 100. Passando al successivo confronto, E27 sarà uguale a 1000 (perché 121 è maggiore di 1,45). La somma finale (E26 + E27), espressa nella cella E29, è equivalente, quindi, a 1100, valore che identifica la presenza di apatite (Fig. 6). Se la somma finale fosse stata di 110 o di 200, avremmo identificato rispettivamente la presenza di brushite o di calcio fosfato (Whitlockite, fosfato octocalcico, ecc.).

Se la somma dei diversi CaP trovati (K24 + K25 + K26) è superiore a 0 (nel nostro caso $127,6 \mu \mathrm{mol} / \mathrm{dL}$ ), il fosforo in eccesso assume il valore di $0,0 \mu \mathrm{mol} / \mathrm{dL}$ (C20), altrimenti viene rappresentato dalla concentrazione di H21 (Fig. 7).

Le diverse quantità di $\mu \mathrm{mol} / \mathrm{dL}$ di brushite, Ca $\mathrm{P}$ e apatite (Fig. 8) sono così rappresentate:

- per la brushite (K24) si considera H21, avendo tale molecola 1 atomo di $P$;

- per il Ca P (K25) si considera (H21-H17)/6,5, avendo tali molecole 6 o 7 atomi di $P$ (media $=6,5$ );

- per l'apatite (K26) si hanno 127,6 $\mu \mathrm{mol} / \mathrm{dL}, \mathrm{H} 21 / 3$, avendo tali molecole 3 atomi di P.

8) Altri contributi per il residuo inorganico: oltre al fosforo già considerato, sono da includere nel residuo inorganico anche gli eccessi di:

- calcio - $0 \mu \mathrm{mol} / \mathrm{dL}$ (D16), ammonio - $0 \mu \mathrm{mol} / \mathrm{dL}$ (D20) e magnesio - 38,68 $\mu \mathrm{mol} / \mathrm{dL}$ (C16).

Se esiste una differenza positiva tra fosforo (H5) e struvite (H17), allora il calcio viene tutto utilizzato per ottenere un $\mathrm{Ca} / \mathrm{P}$ e, pertanto, come nel nostro caso, non dà contributi al residuo inorganico (Fig. 9). 


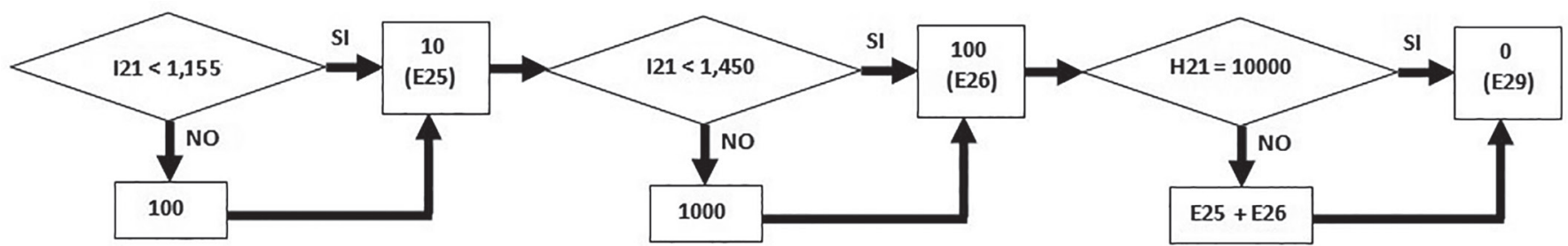

Fig. 6 - Diagramma di flusso per l'identificazione dei diversi calcio fosfato

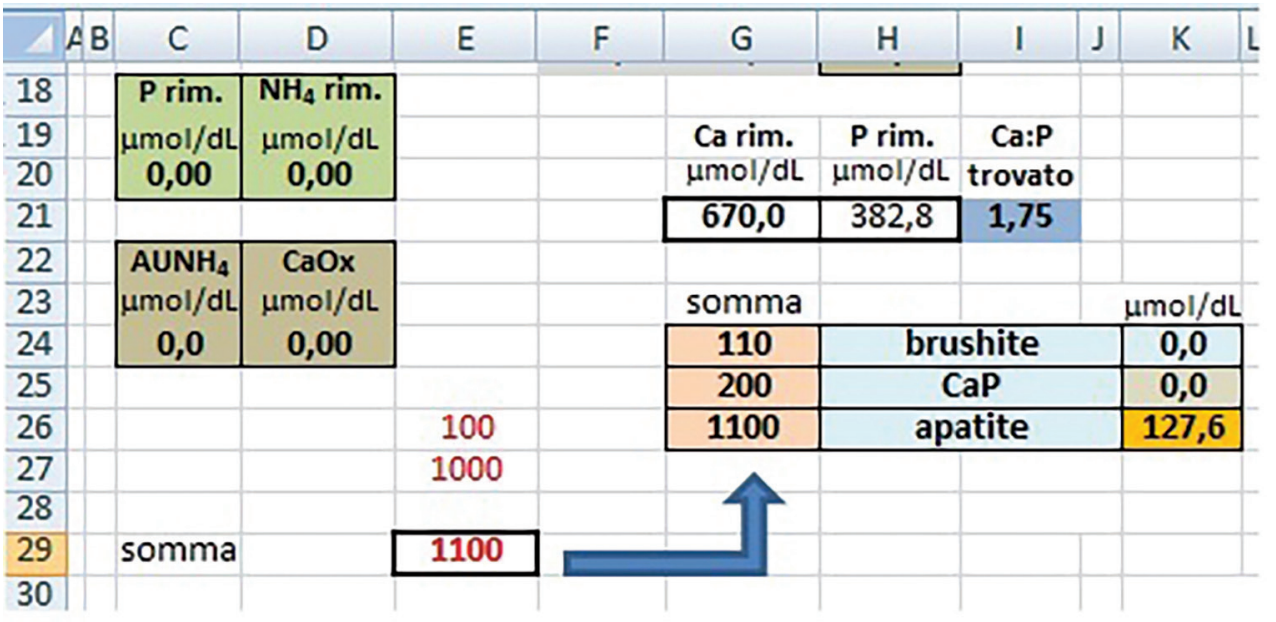

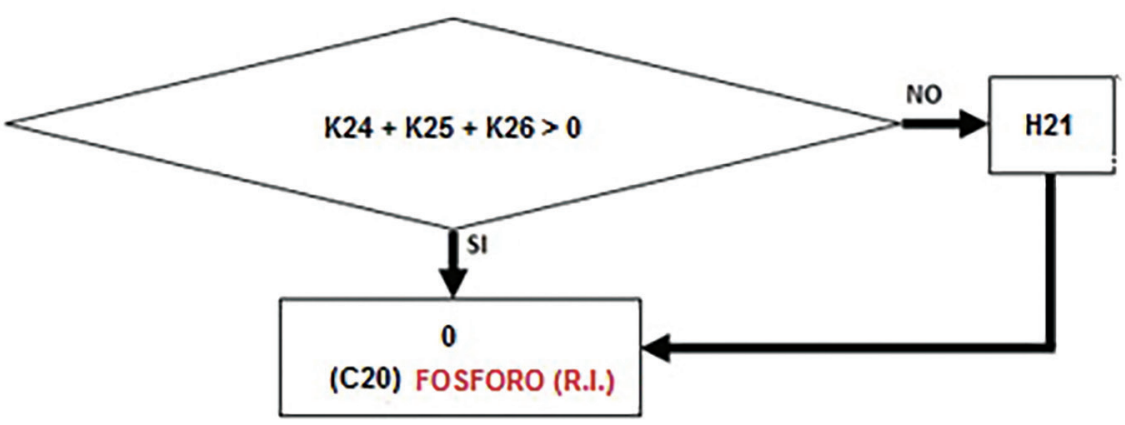

Fig. 7 - Foglio elettronico e diagramma di flusso per la quantificazione del fosforo rimanente

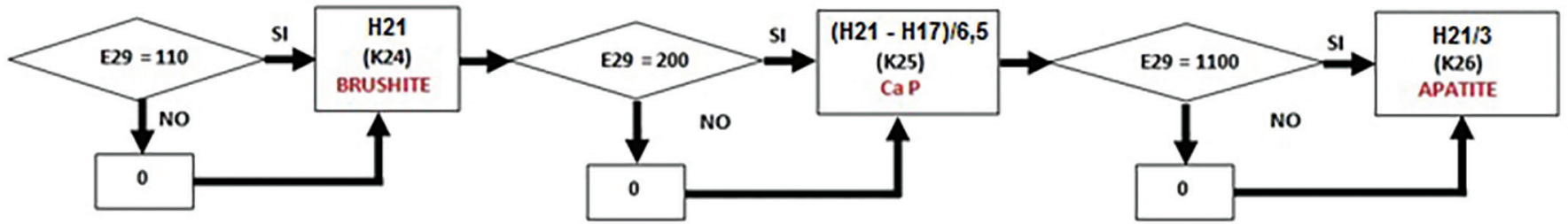

Fig. 8 - Diagramma di flusso per la quantificazione dei diversi calcio fosfato

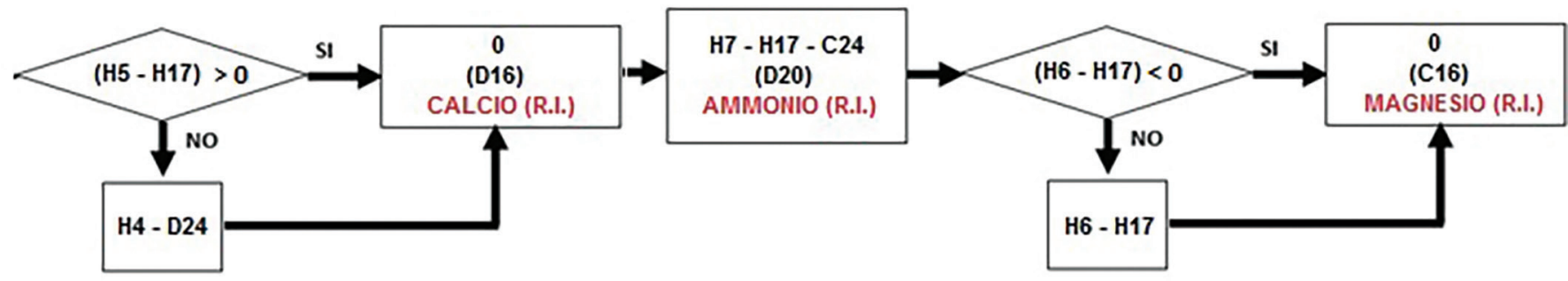

Fig. 9 - Diagramma di flusso per la quantificazione di altri contributi del residuo inorganico 
9) Percentualizzazione delle componenti presenti nel calcolo: la trasformazione in $\mathrm{mg} / \mathrm{dL}$ si effettua moltiplicando le concentrazioni espresse in $\mu \mathrm{mol} / \mathrm{dL}$ per i diversi pesi molecolari (PM) e, precisamente, con la seguente formula: $\mu \mathrm{mol} / \mathrm{dL} * \mathrm{PM} / 1000$.

- I pesi molecolari considerati sono i seguenti:

- N5: CaOx (D24) - 146,1; $\rightarrow 0$ mg/dL

- N6: Struvite (H17) - 245,4; $\rightarrow$ 9,76 mg/dL

- N7: $\mathrm{UANH}_{4}(\mathrm{C} 24)-185,1 ; \rightarrow 0 \mathrm{mg} / \mathrm{dL}$

- N8: Acido urico (H9 - C24) - 168,1; $\rightarrow 0 \mathrm{mg} / \mathrm{dL}$

- N9: Cistina (F10); $\rightarrow 0 \mathrm{mg} / \mathrm{dL}$

- N10: Brushite (K24) - 172,1; $\rightarrow 0 \mathrm{mg} / \mathrm{dL}$

- N11: Ca P (K25) - 1300; $\rightarrow 0$ mg/dL

- N12: Apatite (K26) - 988 (in assenza di carbonato: $\mathrm{E} 12=0) ; \rightarrow 0 \mathrm{mg} / \mathrm{dL}$

- N13: Carbonato Apatite (K26) - 1048,83 (in presenza di carbonato: $\mathrm{E} 12$ = 1); $\rightarrow$ 43,44 mg/dL

- N17: Residuo inorganico: sommatoria dei contributi di magnesio $(\mathrm{C} 16 * 24,3 / 1000=1,53 \mathrm{mg} / \mathrm{dL}$ ), fosforo $(\mathrm{C} 20 * 31 / 1000=0,0 \mathrm{mg} / \mathrm{dL})$, calcio $(\mathrm{D} 16 * 40 / 1000=0,0$ $\mathrm{mg} / \mathrm{dL}$ ) e ammonio (D20*18/1000 $=0,0 \mathrm{mg} / \mathrm{dL}) \rightarrow 1,53$ $\mathrm{mg} / \mathrm{dL}$

- Somma totale dei mg/dL (N18) $\rightarrow$ 145,13 mg/dL

Nelle celle 05-013 viene, poi, effettuata la percentualizzazione delle componenti del calcolo. Nel nostro esempio si ottengono i seguenti risultati: struvite 6,7\% carbonato apatite $92,2 \%$ - residuo inorganico $1,1 \%$.

\section{Discussione}

Il calcolo in questione, analizzato su FTIR della ditta Shimadzu, è riconosciuto in libreria con score abbastanza elevato (Fig. 10). Le componenti rilevate in archivio sono: $90 \%$ di carbonato apatite e $10 \%$ di struvite, molto simili a quelli rilevati in Excel, con il nostro metodo proposto (92,2\% di carbonato apatite $-6,7 \%$ di struvite e $1,1 \%$ di residuo inorganico).

Un altro esempio di determinazione del calcolo, i cui dati biochimici sono rappresentati nel foglio elettronico della Figura 11, evidenzia un risultato sovrapponibile con quello evidenziato all'infrarosso.

Ultimo esempio da proporre viene visualizzato con il foglio elettronico della Figura 12. Anche in questo caso i risultati biochimici sono simili a quelli ricavati dal FTIR della Figura 13. Le componenti ottenute al FTIR sono: $50 \%$ di struvite e $50 \%$ di carbonato apatite, anche in questo caso uguali a quelle estrapolate con Excel (49,6\% di struvite, 50,2\% di carbonato apatite e $0,2 \%$ di residuo inorganico).

Rispetto a un nostro lavoro proposto nel 2016, la nuova procedura di solubilizzazione del calcolo urinario e le opportune diluizioni impostate su Cobas 6000 Roche riducono notevolmente costi e tempi di lavoro.

La possibilità di poter disporre di una metodica al ferrosolfosalicilico per la determinazione degli ossalati, da noi costruita (non presente nel commercio) e adattata su analizzatore biochimico Viva E, potrebbe essere utile in caso di calcoli misti composti in modo preponderante da calcio fosfato e con minore presenza di calcio ossalato.

La metodica al ferro-solfosalicilico è abbastanza rapida e affidabile e, rispetto alla metodica Trinder (quest'ultima sicuramente più precisa ma più costosa), risulta essere scevra da interferenza di fosfati.

II software da noi elaborato in Excel consente di evidenziare le più importanti componenti presenti in un calcolo, inclusa la presenza di alcuni calcio fosfato come brushite e apatite (o carbonato apatite), grazie alla determinazione del rapporto $\mathrm{Ca} / \mathrm{P}$. Valori di quest'ultimo compresi tra 1,155 e 1,450 sono espressi come Ca P (presumibilmente whitlockite, fosfato octocalcico).

L'acquisto dello spettrofotometro all'infrarosso (FTIR), tecnica di riferimento per la determinazione di un calcolo, ha

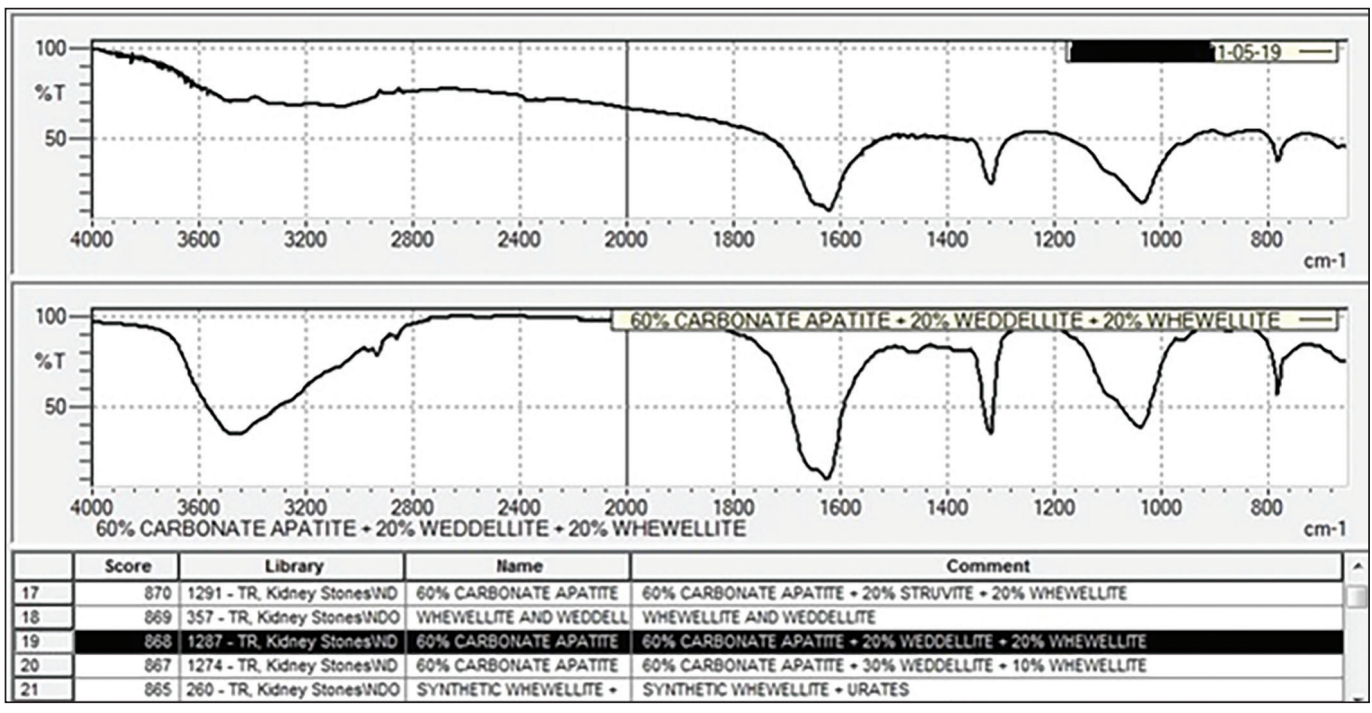

Fig. 10 - Spettro FTIR $60 \%$ carbonato apatite $+20 \%$ weddellite $+20 \%$ wewellite 


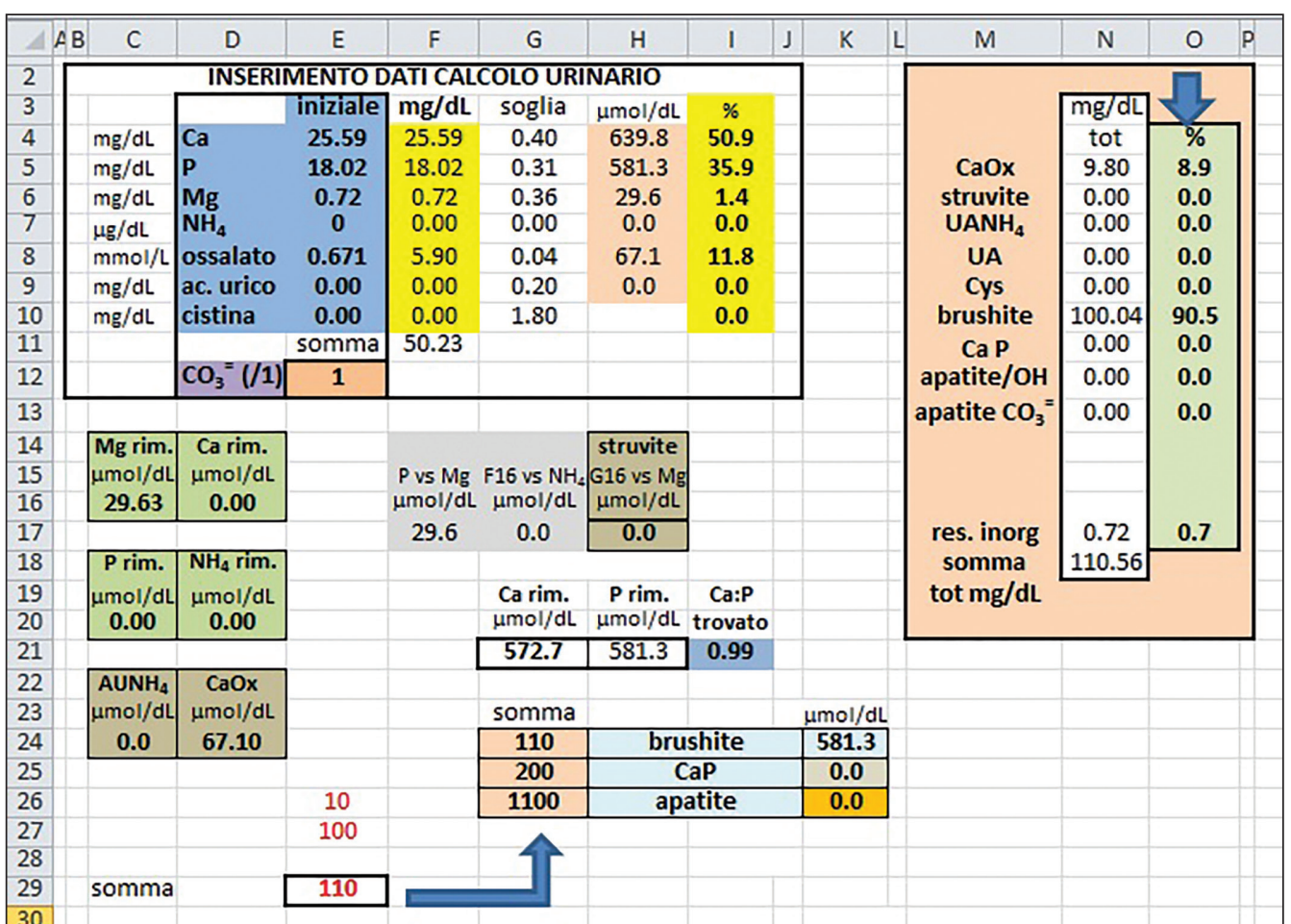

\begin{tabular}{|c|c|c|c|c|c|c|c|c|c|c|c|c|}
\hline$\triangle A$ & C & D & E & $\mathrm{F}$ & G & H & I & J & K & M & $\mathrm{N}$ & 0 \\
\hline \multicolumn{13}{|c|}{ INSERIMENTO DATI CALCOLO URINARIO } \\
\hline 3 & & & iniziale & $\mathrm{mg} / \mathrm{dL}$ & soglia & $\mu \mathrm{mol} / \mathrm{dL}$ & $\%$ & & & \multirow{11}{*}{$\begin{array}{c}\mathrm{CaOx} \\
\text { struvite } \\
\text { UANH }_{4} \\
\text { UA } \\
\text { Cys } \\
\text { brushite } \\
\mathrm{Ca} P \\
\text { apatite/OH } \\
\text { apatite } \mathrm{CO}_{3}=\end{array}$} & $\mathrm{mg} / \mathrm{dn}$ & ᄂ \\
\hline 4 & $\mathrm{mg} / \mathrm{dL}$ & $\mathrm{Ca}$ & 16.59 & 16.59 & 0.40 & 414.8 & 40.6 & & & & tot & $\%$ \\
\hline 5 & $\mathrm{mg} / \mathrm{dL}$ & P & 13.4 & 13.40 & 0.31 & 432.3 & 32.8 & & & & 0.00 & 0.0 \\
\hline 6 & $\mathrm{mg} / \mathrm{dL}$ & Mg & 6.36 & 6.36 & 0.36 & 261.7 & 15.6 & & & & 62.03 & 49.6 \\
\hline 7 & \multirow{6}{*}{$\begin{array}{l}\mu \mathrm{g} / \mathrm{dL} \\
\mathrm{mmol} / \mathrm{L} \\
\mathrm{mg} / \mathrm{dL} \\
\mathrm{mg} / \mathrm{dL}\end{array}$} & $\mathrm{NH}_{4}$ & 4550 & 4.55 & 0.00 & 252.8 & 11.1 & & & & 0.00 & 0.0 \\
\hline 8 & & ossalato & 0.000 & 0.00 & 0.04 & 0.0 & 0.0 & & & & 0.00 & 0.0 \\
\hline 9 & & ac. urico & 0.00 & 0.00 & 0.20 & 0.0 & 0.0 & & & & 0.00 & 0.0 \\
\hline 10 & & cistina & 0.00 & 0.00 & 1.80 & & 0.0 & & & & 0.00 & 0.0 \\
\hline 11 & & & somma & 40.90 & & & & & & & 0.00 & 0.0 \\
\hline 12 & & $\mathrm{CO}_{3}{ }^{=}(/ 1)$ & 1 & & & & & & & & 0.00 & 0.0 \\
\hline 13 & & & & & & & & & & & 62.75 & 50.2 \\
\hline 14 & \multirow{4}{*}{\begin{tabular}{|c|} 
Mg rim. \\
$\mu \mathrm{mol} / \mathrm{dL}$ \\
8.95 \\
\end{tabular}} & \multirow{3}{*}{$\begin{array}{c}\text { Ca rim. } \\
\mu \mathrm{mol} / \mathrm{dL} \\
0.00 \\
\end{array}$} & & \multirow{3}{*}{\multicolumn{2}{|c|}{$\begin{array}{cc}\text { Pvs Mg } & \text { F16 vs } \mathrm{NH}_{2} \\
\mu \mathrm{mol} / \mathrm{dL} & \mu \mathrm{mol} / \mathrm{dL}\end{array}$}} & \begin{tabular}{|l|} 
struvite \\
\end{tabular} & & & & & & \\
\hline 15 & & & & & & $\mathrm{G} 16$ vs $\mathrm{Mg}$ & & & & \multirow{6}{*}{$\begin{array}{l}\text { res. inorg } \\
\text { somma } \\
\text { tot } \mathrm{mg} / \mathrm{dL}\end{array}$} & & \multirow[b]{3}{*}{0.2} \\
\hline 16 & & & & & & $\mu \mathrm{mol} / \mathrm{dL}$ & & & & & \multirow{5}{*}{$\begin{array}{c}0.22 \\
125.00\end{array}$} & \\
\hline 17 & & & & 261.7 & 252.8 & 252.8 & & & & & & \\
\hline 18 & P rim. & $\mathrm{NH}_{4}$ rim. & & & & & & & & & & \\
\hline 19 & $\mu \mathrm{mol} / \mathrm{dL}$ & $\mu \mathrm{mol} / \mathrm{dL}$ & & & Ca rim. & P rim. & Ca:P & & & & & \\
\hline 20 & 0.00 & 0.00 & & & $\mu \mathrm{mol} / \mathrm{dL}$ & $\mu \mathrm{mol} / \mathrm{dL}$ & trovato & & & & & \\
\hline 21 & & & & & 414.8 & 179.5 & 2.31 & & & & & \\
\hline 22 & $\mathrm{AUNH}_{4}$ & $\mathrm{CaOx}$ & & & & & & & & & & \\
\hline 23 & $\mu \mathrm{mol} / \mathrm{dL}$ & $\mu \mathrm{mol} / \mathrm{dL}$ & & & somma & & & & $\mathrm{Imol} / \mathrm{dL}$ & & & \\
\hline 24 & 0.0 & 0.00 & & & 110 & brus & shite & & 0.0 & & & \\
\hline 25 & & & & & 200 & & aP & & 0.0 & & & \\
\hline 26 & & & 100 & & 1100 & & atite & & 59.8 & & & \\
\hline 27 & & & 1000 & & & & & & & & & \\
\hline 28 & & & & & & & & & & & & \\
\hline 29 & somma & & 1100 & & & & & & & & & \\
\hline 30 & & & & & & & & & & & & \\
\hline
\end{tabular}

Fig. 11 - Foglio elettronico 90,1\% brushite $+8,9 \%$ calcio ossalato + $1,0 \%$ R.I.

Fig. 12 - Foglio elettronico 49,9\% struvite $+48,2 \%$ carbonato apatite $+1,9 \%$ R.I. fatto comprendere meglio i limiti di alcuni dosaggi fotometrici usati, il quantitativo di calcolo da pesare e dissolvere in acido solforico, la non facile valutazione dei carbonati e la presenza delle possibili componenti chimiche presenti, specialmente se si tratta di un calcolo misto.
I risultati evidenziati in questo nostro studio preliminare, riferito a 58 calcoli urinari/renali, risultano abbastanza confortanti se paragonati a quelli effettuati con la tecnica di riferimento all'infrarosso (FTIR). Nessun problema è emerso per la determinazione di 19 calcoli costituiti da una singola 


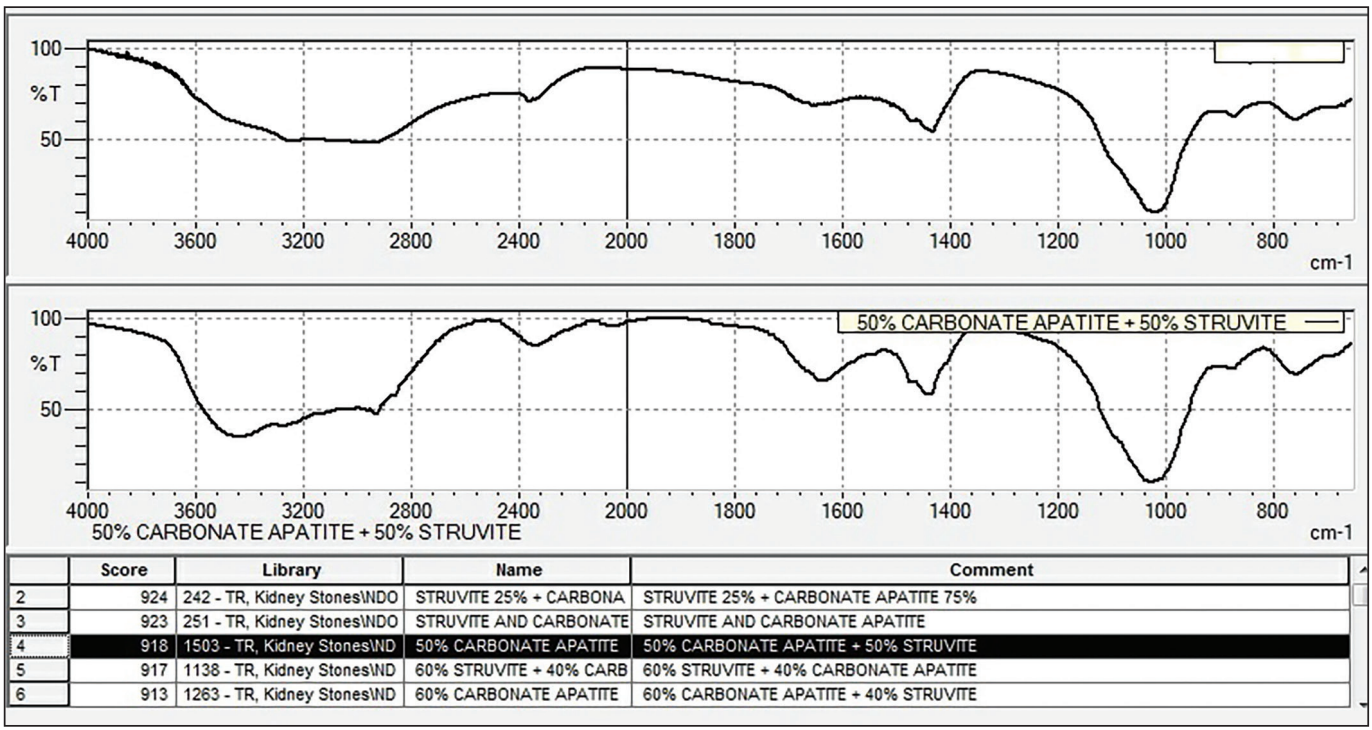

Fig. 13 - Spettro FTIR 50,0\% struvite $+50,0 \%$ carbonato apatite $+1,9 \%$ R.I.

Tabella I - Confronto tra dati excel e dati ftir

\begin{tabular}{lccccc}
\hline $\begin{array}{l}\text { Determinazione biochimica } \\
\text { in Excel }\end{array}$ & $\mathbf{n}^{\circ}$ & $\begin{array}{l}\text { rilev. } \\
\mathbf{C O}_{3}=\end{array}$ & & \multicolumn{3}{c}{ similarità FTIR } \\
\cline { 4 - 7 } & 11 & 6 & 8 & 2 & 1 \\
CaOx + apatite carbonato & 3 & 2 & 2 & 1 & $\uparrow$ \\
Struvite + apatite carbonato & 8 & & 5 & 2 & 1 \\
CaOx + acido urico & 9 & & 6 & 2 & 1 \\
CaOx + brushite & 2 & & 1 & 1 & \\
Acido urico + urato di ammonio & 2 & & & & \\
CaOx + calcio fosfato & 6 & & & &
\end{tabular}

componente, mentre soddisfacenti sono i risultati ottenuti per confronto tra le due tecniche e riferiti a 39 calcoli composti da più di una componente. Non sempre è stato possibile visualizzare la presenza dei carbonati (sviluppo di anidride carbonica con acido minerale), così come segnalato nella Tabella I.

Il software FTIR mostra, invece, delle difficoltà nell'identificazione della composizione di un calcolo misto. Infatti, spesso, vengono riconosciute diverse possibili combinazioni di componenti presenti nel calcolo e, pertanto, ciò non facilita la giusta scelta da parte dell'operatore.

La maggioranza dei campioni di calcolo testati nel nostro studio presenta diverse componenti, espresse in percentuale ed estrapolate in Excel, che sono ben correlate con gli elevati score del FTIR, con valore superiore o uguale a 800 e da noi identificati nella Tabella I con l'espressione $\uparrow \uparrow \uparrow$ (maggiore similarità).

Sono state identificate una media similarità in FTIR ( $\uparrow$ ) per valori di score da 800 a 700 e una bassa similarità ( $\uparrow$ ) per valore di score inferiori a 700 .

Nel nostro Laboratorio si è preferito comunque associare alla determinazione di riferimento anche quella da noi proposta in questo lavoro. Tale scelta ci sembra più che opportuna, al fine di evidenziare il migliore risultato possibile scaturito dalla ricerca della libreria del FTIR. Non sempre infatti è facile capire in FTIR quale siano le reali componenti presenti in calcolo, malgrado possa esserci un alto score di identificazione.

La determinazione del calcolo urinario da noi proposta ha dei costi più che accettabili (inferiore ai 10 euro) ed è facilmente adattabile su strumentazione biochimica, considerando anche i dosaggi dell'ossalato al ferro-solfosalicilico e della cistina praticamente costruiti in laboratorio (determinazioni non commercializzate).

Rispetto all'obsoleto kit in dotazione nella maggioranza dei Laboratori di Patologia Clinica, il metodo da noi proposto è di semplice attuazione e abbastanza affidabile.

Il software da noi creato in Excel facilita l'estrapolazione delle principali componenti del calcolo urinario. Per ulteriori approfondimenti sulla presenza di sostanze poco frequenti, la differenziazione tra wewellite e weddellite e l'identificazione più specifica del calcio fosfato, si rimanda alla tecnica di riferimento FTIR.

\section{Disclosures}

Conflict of interest: The authors declare no conflict of interest. Financial support: This research received no specific grant from any funding agency in the public, commercial, or not-for-profit sectors. Authors contribution: All authors contributed equally to this manuscript.

\section{Bibliografia}

1. Thomas L. Labor und Diagnose. Aufl. Frankfurt: TH-Books Verlagsgeselischaft. 1998;411-5.

2. Textbook of Clinical Chemistry. Ed. by N.W. Tietz, W.B. Saunders Co., Philadelphia. 1999.

3. Tietz Textbook of Clin. Chem. and Molecular Diagnostics. W.B. Saunders Co., Philadelphia. 2012;119:154-581. 
4. Cangiano G, Di Maina E, Buccino G, et al. Estrapolazione in Excel delle componenti del calcolo urinario. Biochimica Clinica. 2013;37:551.

5. Cangiano G, Buccino G, Errico A, et al. Determinazione delle componenti di un calcolo urinario: nuovi accorgimenti metodologici e informatici. Biochimica Clinica. 2016;40:S151.

6. Cangiano G, Latte A, Errico A, et al. Calcolo urinario in Excel. Biochimica Clinica. 2019;43:P110, SS1, S85.

7. Latte $A$, Cangiano $G$, Russo $M$, et al. Screening della cistinuria (metodo al fosfotungstato) su AU600 Olympus e proposta di intervalli di riferimento. Biochimica Clinica. 2005;29:268.

8. Cangiano G, Latte A, Russo M, et al. Determinazione fotometrica della cistinuria. Biochimica Clinica. 2007;31:267-71.

9. Latte $A$, Cangiano $G$, Russo $M$, et al. Determinazione del rapporto cistin/creatinina su AU400 Olympus. Biochimica Clinica. 2007;31:523.

10. Cangiano $\mathrm{G}$, Latte $\mathrm{A}$, Russo $\mathrm{M}$, et al. Dosaggio al fosfotungstato di valori elevati di cistinuria. Biochimica Clinica. 2009;33:491.

11. Cangiano G, Di Maina E, Buccino G, et al. Ottimizzazione del dosaggio fotometrico della cistinuria. Biochimica Clinica. 2013;37:550.

12. Cangiano G, Paradisone C, Di Maina E, et al. Cistinuria al fosfotungstato. Nuovo dosaggio fotometrico a tre reagenti. Biochimica Clinica. 2014;38:460.

13. Cangiano G, Di Maina E, Buccino G, et al. Diagnostica di laboratorio del rischio nefrolitiasico. Biochimica Clinica. 2018;42:327-45.
14. Cangiano G, Buccino G, Gennarelli D, et al. La calcolosi renale in un laboratorio di patologia clinica: modello organizzativo e nuove tecniche analitiche. Giornale di Tecniche Nefrologiche e Dialitiche. 2019;31:22-9.

15. Cangiano $\mathrm{G}$, Latte $\mathrm{A}$, Russo $\mathrm{M}$, et al. Dosaggio degli ossalati urinari su AU 400 Olympus. Biochimica Clinica. 2010;34:536.

16. Cangiano G, Russo M, Forte $F$, et al. Determinazione fotometriche automatizzate di citrato e ossalato urinario. Biologi Italiani. 2010;5/6:21-5.

17. Cangiano G, Latte A, Di Maina E, et al. Ossaluria e cistinuria: dosaggi fotometrici "difficili"... Ma non troppo. Atti del XII Congresso Sez. Campano-Siciliana SIN. 2011;45-9.

18. Cangiano G, Latte A, Di Maina E, et al. Determinazione dei solfati e degli ossalati urinari: ulteriori accorgimenti metodologici. Biochimica Clinica. 2012;36:498.

19. Cangiano G, Buccino G, Errico A, et al. Dosaggio automatizzato degli ossalati presenti in un calcolo urinario. Biochimica Clinica. 2019;43:P180, SS1, S122.

20. Primiano A, Ferraro PM, Persichilli S, et al. Ruolo della spettroscopia infrarossa in trasformata di Fourier nella valutazione della calcolosi urinaria. Biochimica Clinica. 2016;40:28-32.

21. Cangiano $G$, Latte $A$, Di Maina $E$, et al. Informatizzazione di laboratorio del rischio nefrolitiasico. Biochimica Clinica. 2012;36:602.

22. Kelly J. Excel 2003. Guida completa. Editore Apogeo. 2002. 